\title{
Innovation in Fischer-Tropsch: Developing Fundamental Understanding to Support Commercial Opportunities
}

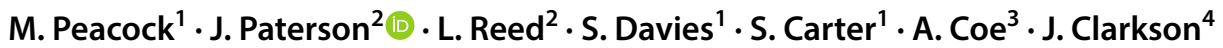

Published online: 12 February 2020

(c) The Author(s) 2020

\begin{abstract}
In this contribution we provide details of the BP-Johnson Matthey proprietary Fischer-Tropsch technology and the advanced CANS reactor and catalyst system. The advanced CANS catalyst carrier reactor provides superior heat transfer, reduced pressure drop and higher productivity that lead to major economic savings. Fundamental understanding of catalyst behaviour is also key to obtaining a catalyst that is stable over the lifetime of its use. Synthesis, calcination and reduction steps introduce changes in the catalyst properties prior to syngas introduction. In particular, the presence of water can affect the final catalyst performance. The activity of a good catalyst can be significantly reduced by a sub-optimal activation or start-up. Similarly, stable operation and minimising deactivation are vital for long and stable catalyst life, with years of operation without requiring regeneration. In this report we also share a fundamental study on the catalyst activation across different catalyst supports. This combines advanced in situ techniques with reactor testing to explore the role of the support on catalyst performance. The results illustrate the critical need for a logical and systematic catalyst development programme to explore these effects to optimise the whole FT process. The combination of a joint approach in development plays a key role in a long term success in a process. The fundamental catalyst understanding, optimisation and improvements in combination with the novel CANS reactor design maximise their potential and offer the potential for a world leading technology.
\end{abstract}

Keywords Fischer-tropsch · Cobalt · CANS reactor · In situ XRD · Activations · Supports

\section{Introduction}

Global energy demands are increasing and so too, is the need for more renewable and alternative sources of energy to help with environmental and energy security concerns. The EU has recently increased their renewable energy target to $32 \%$

Electronic supplementary material The online version of this article (https://doi.org/10.1007/s11244-020-01239-6) contains supplementary material, which is available to authorized users.

\section{J. Paterson}

James.Paterson@uk.bp.com

1 Analytical Group, BP Petrochemicals, DL10, Saltend Chemicals Park, Hull HU12 8DS, UK

2 Centre of Expertise in Applied Chemistry \& Physics, BP, DL10, Saltend Chemicals Park, Hull HU12 8DS, UK

3 Johnson Matthey, 10 Eastbourne Terrace, London W2 6LG, UK

4 Johnson Matthey, Princeton Drive, Stockton-on-Tees TS17 6PY, UK for 2030 [1], and many countries are also looking at reducing waste and other circular economy concepts. However, the transportation industry is one of the most challenging sectors to adapt to using low-carbon fuels. Transportation modes such as aircraft, heavy-duty and marine vehicles demand high power and energy capacity that are currently unmet by renewable technologies. In the interim, we need clean, sustainable methods, continuous improvement and new innovations in renewable fuels to meet EU targets. The new BP/Johnson Matthey CANS technology presented here shows how catalyst development in combination with process innovation can give a commercially viable technology process for synthetic (bio)fuels. These developments have come from years of fundamental research in combination with a strong understanding of the critical factors affecting Fischer-Tropsch chemistry. A summary of this work is shown here with the benefits of the commercial CANS technology and some examples of how catalyst understanding and evaluation can be critical to the final performances.

Typical Fischer-Tropsch processes (Fig. 1) utilize a syngas feed from bio or fossil fuels and convert to hydrocarbon 


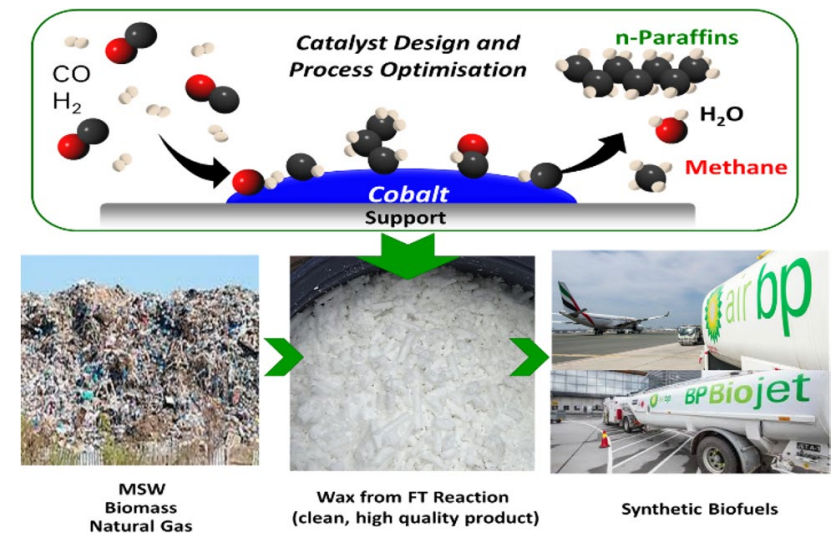

Fig. 1 Fischer-Tropsch process for syngas to paraffins over a cobalt catalyst plays a vital role in an overall waste to fuels process

products (Eq. 1). Upgrading the wax product by hydrotreating and cracking makes a high-quality fuel for transport or specialty oils/wax products.

Industrial research at companies such as BP and Johnson Matthey develop catalytic processes through focusing research on understanding the fundamental effects that influence commercial performance. Understanding the catalyst design, treatments and long-term operation is at the forefront of the BP research centre activities with careful application of in situ or in operando X-ray techniques to demonstrate the impact of the catalyst's environment [2,3]. Observing catalyst behaviour under realistic process conditions (suitable for commercial scale up), is crucial to understanding how the catalyst evolves with time in a fixed bed or slurry type reactor. An example of this is in Fischer-Tropsch (FT) chemistry, where an oxidic (calcined) catalyst is loaded into a fixed bed reactor and reduced in hydrogen to form the active catalyst, before switching to syngas $\left(\mathrm{CO} / \mathrm{H}_{2}\right)$ and operated for thousands of hours. A consequence of this process is the need to understand the evolution and catalytic changes as the plant continues to operate. This is necessary for several reasons including risk management and continued improvements to increase productivity for the commercial operation.

$(2 \mathrm{n}+1) \mathrm{H}_{2}+\mathrm{nCO} \rightarrow \mathrm{C}_{\mathrm{n}} \mathrm{H}_{2 \mathrm{n}+2}+\mathrm{nH}_{2} \mathrm{O}$

$\mathrm{CO}+\mathrm{H}_{2} \mathrm{O} \leftrightarrow \mathrm{CO}_{2}+\mathrm{H}_{2}$

$2 \mathrm{CO} \rightarrow \mathrm{CO}_{2}+\mathrm{C}$

$2 \mathrm{CO}+\mathrm{Co} \rightarrow \mathrm{Co}_{2} \mathrm{C}+\mathrm{CO}_{2}$

Catalyst development requires investigation across multiple treatments, processes and scales (Fig. 2) [4]. The catalyst synthesis is often crucial to the resulting performance - use of promoters, order of reagent addition, choice of supports

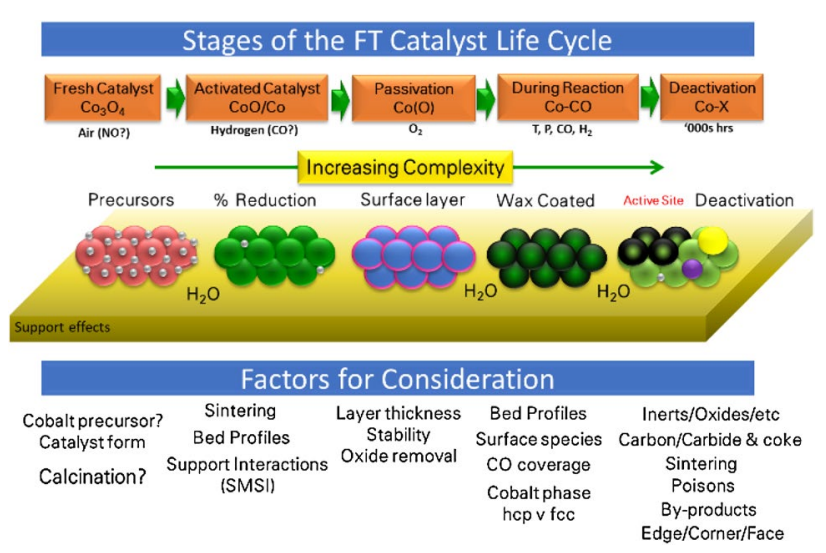

Fig. 2 Stages of the evolution of the catalyst during operation (1-r) catalyst synthesis, activation, passivation, operation and deactivation all play a vital role in catalyst performance

and preparation conditions all influence the catalyst behaviour [5]. Catalyst activation (Eqs. 5-7) in hydrogen or another reducing gas leads to changes in cobalt phases and water production which impacts on the degree of reduction, sintering and fixed bed activity profiles [6].

$\mathrm{Co}_{3} \mathrm{O}_{4}+\mathrm{H}_{2} \rightarrow 3 \mathrm{CoO}+\mathrm{H}_{2} \mathrm{O}$

$3 \mathrm{CoO}+3 \mathrm{H}_{2} \rightarrow 3 \mathrm{Co}+3 \mathrm{H}_{2} \mathrm{O}$

Overall:

$\mathrm{Co}_{3} \mathrm{O}_{4}+4 \mathrm{H}_{2} \rightarrow 3 \mathrm{Co}+4 \mathrm{H}_{2} \mathrm{O}$

Subsequent catalyst passivation in dilute oxygen is often used to give a catalyst that is suitable for analysis, but we have found this is often unstable and the passivated catalyst can change with time/conditions [7, 8]. Finally, FT catalysts are used under high temperatures and pressures with varying partial pressures of hydrogen and carbon monoxide [9]. The FT reaction produces a wide range of products including water, which can cause catalyst transformations and deactivation. Syngas partial pressures decrease down the bed which can lead to local changes in the $\mathrm{H}_{2}$ : $\mathrm{CO}$ ratio and thus to extreme conditions or the water-gas-shift reaction (WGS, Eq. 2). Catalyst deactivation has been shown in the literature $[10,11]$ to be caused by a range of mechanisms including: sintering, coke formation (Eq. 3), carbide formation (Eq. 4), oxidation and inert support species as shown below. BP \& Johnson Matthey have chosen to avoid the most harsh conditions for operation, which often produce better selectivity, in order to control and reduce catalyst deactivation. The novel advanced CANS technology enables these high selectivity's to be achieved. It is well known in the field that low syngas ratios (less than approximately 1.4) lead to 
carbon lay down and coking at the back of the bed where the ratio is well below 1. Likewise, high conversion and highwater partial pressures have been avoided to reduce sintering and catalyst oxidation. Alumina catalyst supports commonly form cobalt aluminates which are not catalytically active for FT, while gas clean-up is strongly controlled to avoid the build-up of catalyst poisons. While the exact mechanism is unclear, published work has shown a link between the partial pressures of hydrogen and water and cobalt aluminates via a cobalt oxide intermediate. High $\mathrm{H}_{2} \mathrm{O}: \mathrm{H}_{2}$ levels can lead to some cobalt metal in the catalyst being oxidised to $\mathrm{CoO}$ and this in turn is the precursor for forming cobalt aluminates/ titanates/silicates $[12,13]$. Regenerations have been shown [14] to be beneficial to regain catalyst activity, but it should be noted that this is often introduces additional risk, requires significant capital expenditure (CapEx) commercially and is not always suitable for the mode of deactivation seen. The main regeneration discussed in the public literature is via an oxidation and re-reduction treatment, whereby an oxidation of the catalyst is used to break up sintered cobalt particles and re-disperse across the catalyst.

In this contribution we share the concepts of our new advanced reactor system and the benefits over conventional FT processes. This highlights the role that pressure drop, heat transfer and catalyst size have on commercial designs. The new reactor design enables high selectivity, high productivity and long term operational/catalyst stability at scale. It combines the desirable properties of fixed bed reactors with the benefits of slurry FT processes to give an economic, modular and a high-performance alternative to current options. We also highlight the benefit of detailed catalyst understanding in reactor testing through the evaluation of cobalt supported catalysts across a range of metal oxide supports. This highlights the critical need for chemical understanding in catalyst synthesis, activation and testing to explore a catalyst development programme. In situ characterization via XRD enables clear understanding of the cobalt oxide reductions on different supports, and the role a support plays in controlling performance in FT testing.

\subsection{BP-JM Technology Development}

Observing catalyst behaviour under realistic process conditions (suitable for commercial scale-up) is critical to understanding how the catalyst evolves over time in a fixed-bed or slurry-type reactor. BP and Johnson Matthey's development of Fischer-Tropsch dates to the early 1980s [15, 16] and the development of a commercial demonstration of the technology between 2002 and 2009 at Nikiski (Fig. 3). The integrated plant combined three processes for testing Fischer-Tropsch technology: a proprietary compact reformer for syngas generation, fixed bed Fischer-Tropsch reactor, and mild hydrocracking of Fischer-Tropsch waxes to

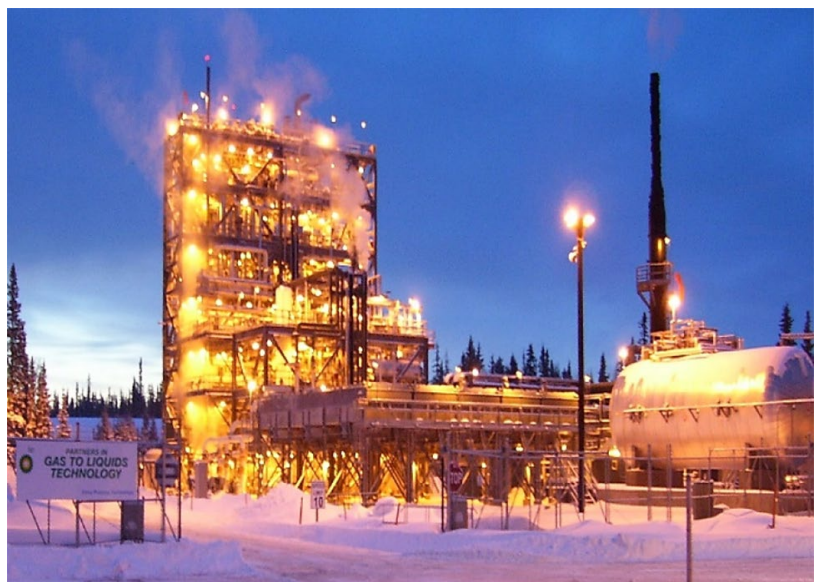

Fig. 3 The Nikiski Demonstration Plant in Alaska, which produced $300 \mathrm{bbl} /$ day of synthetic fuels product

produce synthetic crude. The original fixed bed tubular reactor technology was Developed as a method of monetising stranded natural gas in remote locations. However, it was only competitive at large scale $(>30,000 \mathrm{bbl} / \mathrm{d})$ in areas with low natural gas prices and high oil prices.

More recently the BP partnership with Johnson Matthey Davy has developed an advanced catalyst and fixed bed reactor design employing CANS catalyst carriers [17]. This has shown significant benefits over other commercially available GTL technologies for selectivity and activity. This allows powder-like catalysts with minimal mass transfer resistance to be used in a fixed-bed reactor system for improved selectivity ( $>90 \% \mathrm{C}_{5+}$ selectivity on commercial particles). High heat transfer enables coupled with low pressure drop enables high productivities $\left(>300 \mathrm{kgC}_{5+} / \mathrm{m}^{3}\right.$ cat $\left./ \mathrm{h}\right)$ and an increasing yield per unit volume of reactor and reduced reactor footprint. This technology is now being commercialised for a waste to fuels project in the USA and will commence production in 2020 [18].

Careful control of cobalt crystallite size $[19,20]$ is vital to ensure optimal selectivity throughout the life time of the catalyst. Small cobalt particles $(<6 \mathrm{~nm})$ favour termination due to the excess of edges and the slow $\mathrm{C}-\mathrm{O}$ dissociation, which is the rate determining step in FT. Large cobalt crystallites increase bulk phase cobalt with a reduction in active surface area, requiring higher temperatures to achieve a target conversion. Diffusion of syngas in larger extrudate pellets (which are required for conventional fixed bed reactors) introduces detrimental selectivity effects as $\mathrm{CO}$ and $\mathrm{H}_{2}$ diffuse into the catalyst pores (Fig. 4). The novel CANS reactor design combines the advantages of the fixed bed tubular reactors with slurry phase systems. Its modular design enables scalable and operationally simplistic Fischer-Tropsch synthesis while the smaller catalyst particles offer high productivity and selectivity (Figs. 4, 5). The 


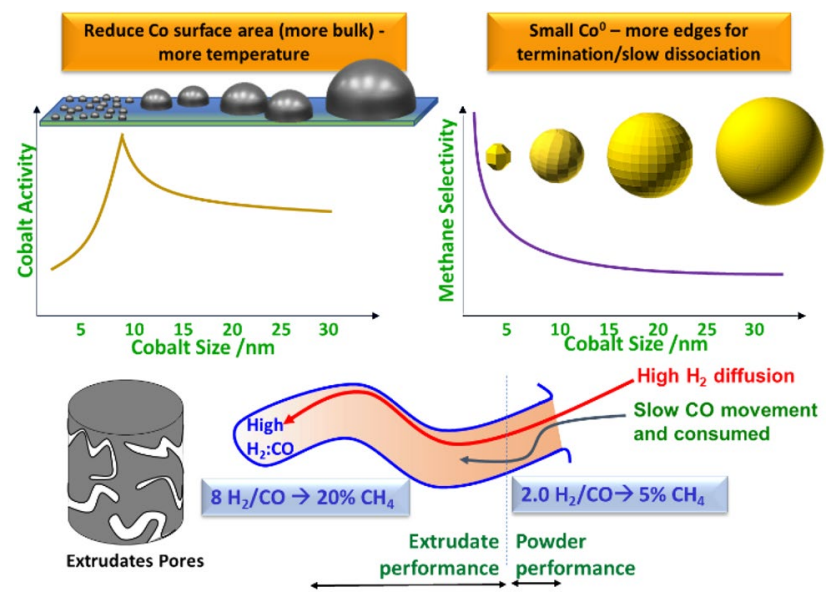

Fig. 4 Effect of cobalt particle size on FT activity and selectivity (top) and Effect of syngas diffusion rates on selectivity in extrudate pellets versus powders (bottom)

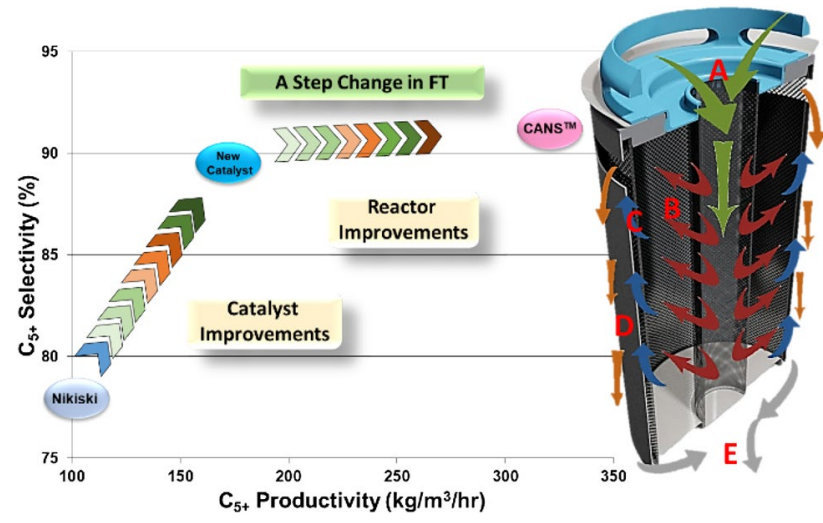

Fig. 5 The change from the first-generation technology (Nikiski) to a new generation 2 catalyst offered an improvement in hydrocarbon selectivity, while reactor improvements led to a step change in productivity. Inset-a schematic of the catalyst carrier with gas flow paths

stacked catalyst carriers have a unique design that aids their ability to perform Fischer-Tropsch synthesis as shown in Fig. 5. Syngas arrives from the catalyst carrier above and travels down a porous central channel (A), flowing radially through the catalyst bed where the Fischer-Tropsch reaction occurs and heat is evolved (B). The gas exits via a porous outer wall, flowing towards the top inner side of the catalyst carrier body $(\mathrm{C})$. Cooling occurs as the gas flows down the narrow annulus between the body and inside wall of the tube, through the transference of heat to boiling water on the shellside (D). A seal prevents gas bypassing the next catalyst carrier and the gas then enters the catalyst carrier below with the process then repeated $(\mathrm{E})$.

A reactor tube contains $60-80$ CANS catalyst carriers and effectively creates mini-adiabatic radial flow reactors with intercooling. The radial flow through each catalyst carrier enables the use of smaller catalyst particles which provide improved selectivity and activity. Pressure drop $(\Delta \mathrm{P})$ across the reactor is carefully optimised such that the effective catalyst bed length is reduced to $2-3 \mathrm{~m}$ rather than a conventional 10-15 m commercial fixed bed. The effective catalyst bed thickness is only around $15 \%$ of the overall tube length. These factors enable smaller catalyst particles to be used, operating at higher productivity and better selectivity. The novel design therefore allows for sub-millimetre catalyst particles, compared with the 1-4 mm catalyst pellets used in traditional fixed bed reactors. The wide diameter of the tubes reduces the heat transfer surface per unit volume of catalyst; this is compensated by a larger temperature difference at the wall where reactants are hottest. The CANS catalyst carrier design turns the heat transfer profile inside out such that the hottest point (after exothermic catalyst bed) is next to the cool tube wall (Fig. 6). Combining this structure with a high gas velocity between the catalyst carrier body and tube wall results in excellent heat transfer. By separating heat removal from the catalyst bed, good control of the reaction temperature is also achieved without the risk of quenching the reaction. The modular design of a CANS catalyst carrier enables greater flexibility in commercial applications. CANS catalyst carriers can be loaded with different catalysts at the top or the bottom to address any performance deficiency. This can extend to different cobalt loadings, catalyst particle sizes, cobalt crystallite sizes and intrinsic activity. Similarly, a different catalyst could be used, such as a traditional FT catalyst in the top CANS carriers and a zeolite or hydrocracking catalyst in the lower CANS to combine FT and upgrading. Similarly the top CANS carriers could contain gas clean-up/guard beds or a shift catalyst to adjust the syngas ratio entering the FT catalyst CANS carriers. The CANS catalyst carrier dimensions, central channel diameter, catalyst bed depth and height, inner/outer annulus width can be tuned to the process or catalyst activity such that CANS carriers with different annulus or catalyst bed sizes can be used in different combinations of CANS carrier dimensions can be used through the tube [21].

Compared with conventional fixed bed tubular reactors, the new catalyst carriers and optimised catalyst reduces CapEx costs by around $50 \%$ and the number of reactor tubes by $95 \%$ due to the larger tube diameter and increased productivity. There is also a threefold increase in production for the same size reactor as the catalyst performance is closer to that of a powder, with excellent heat and mass transfer to and from the catalyst particles. The increased productivity at least halves the catalyst volumes usually required for the same production rate. Furthermore, containing the catalyst inside the carrier removes the requirement to filter the catalyst from the wax product. Instead the catalyst is easily separated by removing the entire catalyst carrier meaning there 
A
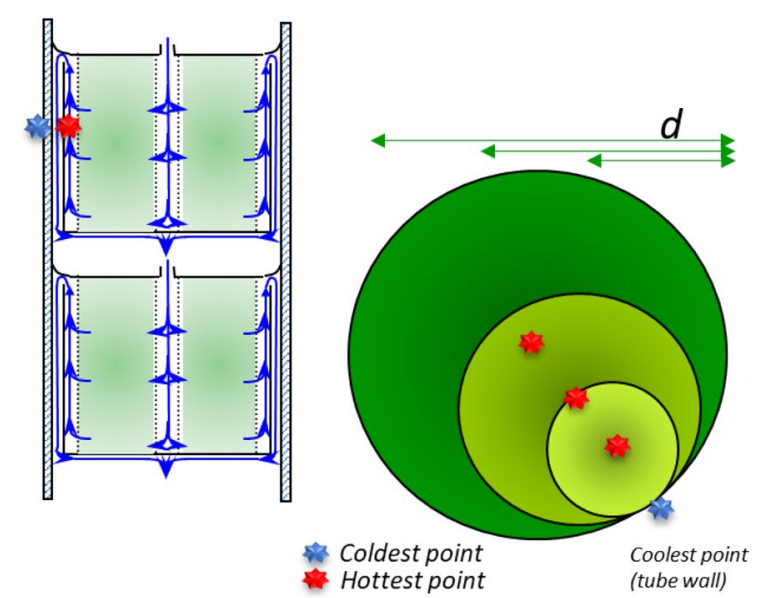

Operate at higher productivity with fewer tubes

B

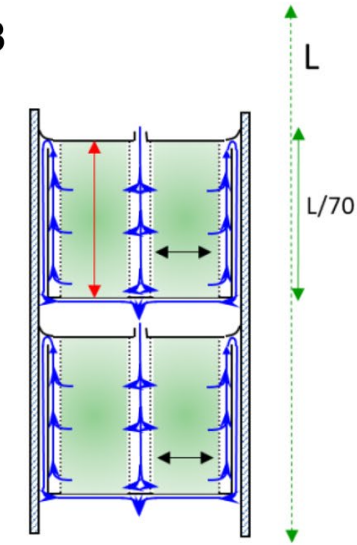

$\triangle P$ across $2-3 m$ of catalyst (sum of black arrows, not red arrows)

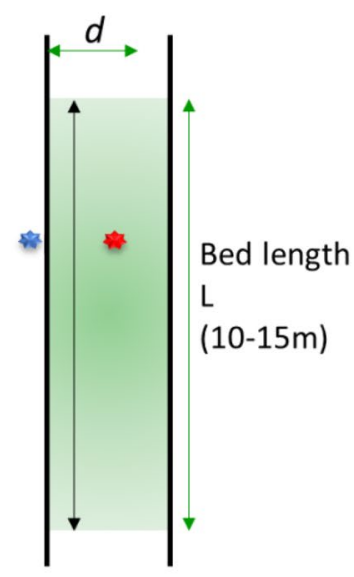

$\Delta P$ across $10-15 m$ of catalyst

Operate with smaller catalyst particles with better selectivity

Fig. 6 Benefit of CANS Catalyst Carriers for heat transfer and commercial tube pressure drop

is no interaction with the hazardous cobalt catalyst material. Fundamentally, this makes Fischer-Tropsch applications possible at both small and large scales, with around 6000 barrels per day achievable in a single reactor of around 900 tonnes. For areas with tighter transport restrictions, 2000 barrels per day can be delivered in a single reactor of around $4 \mathrm{~m}$ diameter and 250 tonnes in weight.

In this contribution, we discuss the role of catalyst synthesis and activation for Fischer-Tropsch, using realistic conditions and in situ characterization. FT catalyst performance depends on a great number of factors, with support effects during activation being critical to achieving good selectivity and activity. Here we cover a range of supports for catalysts including BP's first-generation catalyst though to the current generation coupled with CANS catalyst carrier technology as well as other interesting supports for FT based chemistry.

\section{Experimental}

\subsection{Catalyst Synthesis}

Catalysts were prepared by impregnation of a cobalt nitrate hexahydrate (98-102\%, VWR from Alfa Aesar) and manganese acetate tetrahydrate (99\% Aldrich) aqueous solution on to the support materials. The support materials were received from St Gobain (alumina, titania \& zirconia), VWR (zinc oxide), ceria (Aldrich) and silica (Evonik). Catalyst were all prepared in a similar way, with cobalt and manganese precursors dissolved in a minimal amount of water and warmed at $40{ }^{\circ} \mathrm{C}$ for $10 \mathrm{~min}$. During dissolving of the precursors an endothermic dissolution process meant the solution became cold and the $40{ }^{\circ} \mathrm{C}$ oil bath helped to speed this process up. The resulting solution was added to the support materials and mixed until a homogeneous material was produced. The materials were dried at $120^{\circ} \mathrm{C}$ and calcined at $300{ }^{\circ} \mathrm{C}$ for $3 \mathrm{~h}$. In the case of the zinc oxide support a reactive synthesis occurred during impregnation, which is due to cobalt interacting with $\mathrm{ZnO}$ to form a mixed metal spinel. The colour of the mixture changed from light pink to purple and the texture hardened until a cement like material was left. This hard cement was transferred to a crucible for calcination in line with the other samples.

\subsection{XRD}

Powder X-ray diffraction (pXRD) is a technique commonly used to analyse solid catalyst samples to provide structural information about the crystalline phases present in a catalyst, as well as crystallite size and relative crystalline phase composition via such methodologies as Rietveld Refinement. The pXRD was carried out by grinding the catalyst pellets to a fine powder by hand using a pestle and mortar, and the powder is then front loaded in to a standard Bruker powder diffraction sample holder. Data is collected using Bragg Brentano geometry with copper radiation at $1.6 \mathrm{kV}$ and a LynxeyeXE position sensitive detector. This is optimised to reduce fluorescence over the 2-theta range of $10^{\circ}-100^{\circ}$, whilst rotating the sample to improve counting statistics. Crystalline phases are identified by search matching within the powder diffraction file provided by the International Centre for Diffraction Data (ICDD). Rietveld analysis is carried out within Topas v4.3, with the instrumental broadening factors established from a vendor supplied corundum standard and NIST sourced $\mathrm{LaB}_{6}$ over the same scan conditions. 


\subsection{In Situ XRD}

The in situ XRD conditions utilise the same sample preparation as pXRD. However, the catalyst is loaded into the open sample holder of an XRK900 Anton Paar reaction chamber, Fig. 7 [6]. The amount of powder used in the test is enough to fill the sample holder which is approximately $150 \mu \mathrm{l}$ in size. Reactive gases are controlled using thermal mass flow controllers to supply $50 \mathrm{ml} / \mathrm{min}$ to the chamber. The open sample holder is configured so the gas flooding into the chamber must exit by passing through the relatively loosely packed powder. This ensures intimate contact of the reactive gases and the sample, without relying on gas diffusion. The instrument is configured in a theta/two-theta format, with the high-powered TXS-Cu anode in a fixed position, the sample moving by theta and the Lynxeye detector moving by twotheta. Data is collected at $10.5 \mathrm{kV}$ over the range of $15^{\circ}-90^{\circ}$ two-theta with a fixed divergence slit set at $0.3^{\circ}$, a step size of $0.022^{\circ}$ and a count time of $0.9 \mathrm{~s}$ per step. The temperature ramp between CANS was carried out at $40^{\circ} \mathrm{C} / \mathrm{h}$, with the initial pattern collected at $150{ }^{\circ} \mathrm{C}$. The subsequent CANS were collected every $5{ }^{\circ} \mathrm{C}$ which slows the overall temperature ramp down between 150 and $300{ }^{\circ} \mathrm{C}$ as the temperature is static during the collection.

\subsection{Temperature Programmed Reduction TGA-TPR}

Thermo-gravimetric analysis (TGA) is used to screen the reduction of metal oxide phases by flowing dilute hydrogen over the sample. The dilute hydrogen gas, $4 \mathrm{~mol} \% \mathrm{H}_{2}$ in $\mathrm{Ar}$, is used to avoid a high concentration of $\mathrm{H}_{2}$ forming a potentially flammable atmosphere. Also flowing through the TGA is a protective cover of $\mathrm{N}_{2}$, effectively producing $\mathrm{a} \mathrm{H}_{2}$ concentration of $2 \mathrm{~mol} \%$. The samples are heated at a ramp rate of $2{ }^{\circ} \mathrm{C} / \mathrm{min}$ from 30 to $800{ }^{\circ} \mathrm{C}$ and the mass of the sample monitored continuously to $6 \mathrm{~d}$.p of a gram. The loss of oxygen during the reduction is observed by the reduction in mass of the catalyst sample from the formation of water.

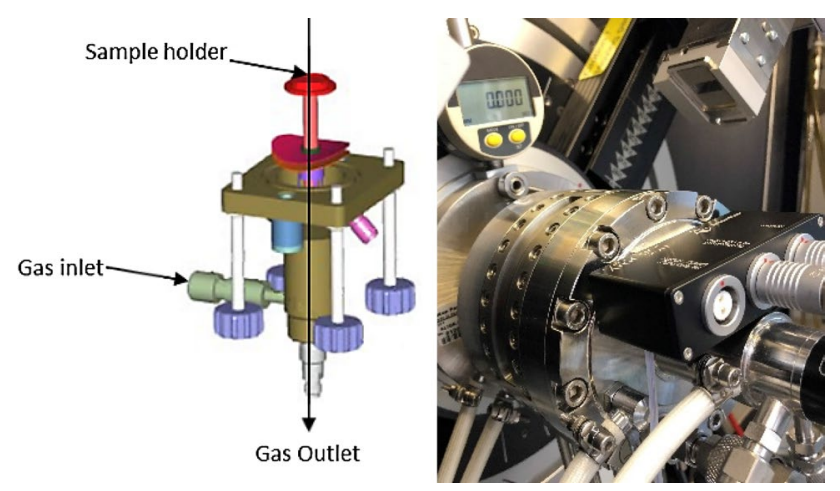

Fig. 7 Showing the sample holder configuration of the XRK-900 chamber, with gas flow through the catalyst bed

\subsection{Elemental Analysis}

The cobalt and manganese concentration in the catalysts was determined by methodologies dependent upon the support material of the catalyst.

Catalysts supported on ceria and zinc oxide were analysed by digestion of the material using a mixture of sulphuric and nitric acid, with the resulting solution made up to a standard volume with UHQ water and subsequently analysed by ICP-OES.

The catalysts made on titania, alumina \& silica were all analysed by XRF. The catalysts were fused with a mixture of lithium tetraborate, lithium metaborate and flux at $1050{ }^{\circ} \mathrm{C}$ before being cast into a $32 \mathrm{~mm}$ glass bead. Calibration of the XRF was made by synthesising standards from pure oxide materials using the same preparation method as the catalyst samples.

The Zirconia sample was digested on a hot plate at $350{ }^{\circ} \mathrm{C}$ with a 5:2 ratio of $\mathrm{H}_{2} \mathrm{SO}_{4}:\left(\mathrm{NH}_{4}\right)_{2} \mathrm{SO}_{4}$ for $3 \mathrm{~h}$. The resulting solution was made up to a standard volume using UHQ water and subsequently analysed by ICP-OES.

\subsection{Reactor Testing}

Catalysts were tested in a high throughput reactor with a single heating block, using $100 \mathrm{mg}$ of catalyst in SiC diluent. Catalyst activation was completed at $8000 \mathrm{~h}^{-1} \mathrm{GHSV}$ in $100 \% \mathrm{H}_{2}$. A temperature ramp of $80^{\circ} \mathrm{C}$ to $150{ }^{\circ} \mathrm{C}$ at $2{ }^{\circ} \mathrm{C} /$ min and $150{ }^{\circ} \mathrm{C}$ to $300^{\circ} \mathrm{C}$ at $1{ }^{\circ} \mathrm{C} /$ min was used, with a $15 \mathrm{~h}$ dwell at $300{ }^{\circ} \mathrm{C}$. Fischer-Tropsch tests were carried out at $30 \mathrm{barg}$ at $3000 \mathrm{~h}^{-1} \mathrm{GHSV}$ and $1.8 \mathrm{H}_{2}: \mathrm{CO}$. Unit temperature was adjusted in the unit to a reasonable conversion across the liners. High throughput testing is a valuable technique for high data quality and testing under identical conditions. In this work a 16-fold reactor was used with all liners loaded and tested simultaneously to give reliable data. All catalyst positions were at a common temperature and pressure and all had a common gas feed. Online GC analysis was done in sequence around the 16 liners to give time on stream data.

\section{Results and Discussion}

\subsection{Basic Lab Characterisation on Fresh Catalyst, $\mathbf{N}_{2}$, Elemental}

The elemental analysis of the fresh catalysts shows consistent cobalt loading throughout the sample set with an average of $10.7 \%$ cobalt and $1.2 \%$ Manganese on the samples. These are all close enough to one another to provide useful comparison during catalytic testing.

There are some very clear differences between the surface area of the six supports being studied, with silica and 
alumina both having very high surface area, titania and zirconia have an intermediate surface area and the zincite and ceria have a very low surface area. Sulphur was present on the plain silica support as received from suppliers. Sulphur is a well-known poison for cobalt FT and as such the performance in reactors was negligible, but it does provide a good reference catalyst for XRD analysis.

\subsection{TGA-TPR}

The TGA reduction of the catalysts under dilute hydrogen shows the sequential changes in oxidation state of the cobalt oxide spinel. Some additional peaks were observed with low temperature $\left(100-200{ }^{\circ} \mathrm{C}\right)$ mass loss associated with removal of water and/or residual nitrate decomposition from the precursors. The peaks at $250{ }^{\circ} \mathrm{C}$ and $450{ }^{\circ} \mathrm{C}$ relate to the reduction of cobalt oxide spinel to cobalt metal via cobalt monoxide, Fig. 8 (Eq. 5-7).

There are other notable differences between the reduction behaviour of the catalysts between this sample set. The gamma alumina supported catalyst requires very high temperatures to reduce the spinel in the dilute hydrogen of the TGA experiment. Indeed, this may not be fully reduced, and only modest mass loss was observed even during the high temperature mass loss at $700{ }^{\circ} \mathrm{C}$. The reduction of the spinel to the monoxide is also higher than the rest of the catalysts, with the peak mass loss above $300{ }^{\circ} \mathrm{C}$. The ceria supported catalyst reduction of the spinel phase to the monoxide phase and the subsequent reduction of the monoxide to the metal are not fully resolved. This suggests the cobalt monoxide may be reducing to metal before all the spinel has been reduced to the monoxide phase (both reduction stages occurring together). The reduction of $\mathrm{CoO}$ to the metal with the ceria sample

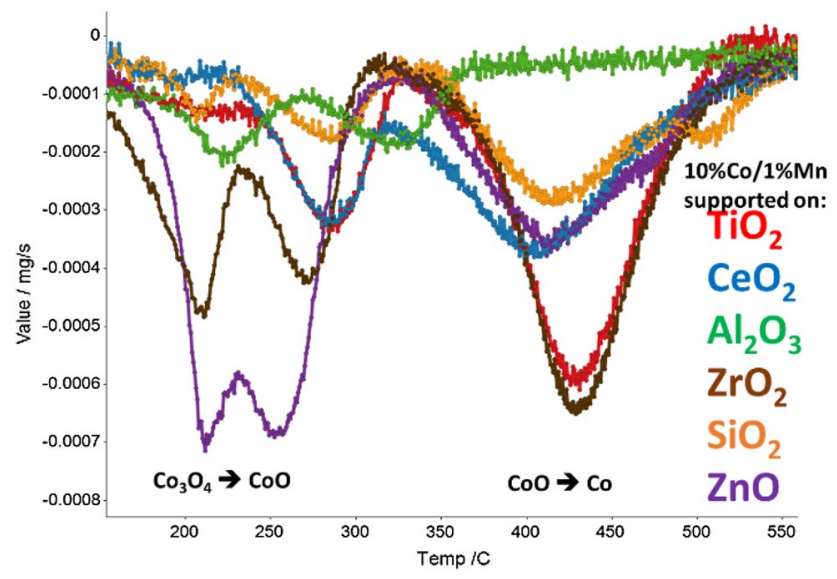

Fig. 8 TPR reduction differential profiles of the catalysts used in the in situ XRD study, showing the 2-stage reduction of cobalt oxide to cobalt metal starts at the lowest temperature of this set of catalysts, despite the reduction of the spinel occurring at the higher temperature than all other catalysts except for the gammaalumina supported catalyst.

\subsection{X-ray Diffraction (XRD)}

The diffraction analysis of the fresh materials all show the cobalt exists in a spinel structure of a mixed oxidation state oxide. On the most part this spinel phase looks quite similar between the six different samples, with the clearest differences existing for the zincite supported catalyst and the ceria supported catalyst as observed in Fig. 10. The spinel peaks for the ceria supported catalyst are somewhat narrower than for the other catalysts, due to a larger crystallite size of the spinel phase, as indicated in Table 1. The crystallite size of the spinel phase for all the other catalysts is in the range of $54-75 \AA$, whereas the ceria supported sample shows a crystallite size of $121 \AA$. The zincite supported catalyst is the other catalyst to exhibit differences in the spinel phase. Whilst the crystallite size of the spinel is in a similar range to most of the catalysts here, the peak position on the spinel phase is shifted significantly, as can be seen in Fig. 10. This is due to the incorporation of zinc into the structure, giving rise to the mixed metal spinel which is causing an increase in the unit cell size and the shift in peak position within the diffraction pattern. Cobalt nitrate addition to zinc oxide powder undergoes a spontaneous reaction to form the mixed metal spinel [22], and this changes colour from pink to purple as a result. The catalyst preparation is also different, with the paste/mixture of $\mathrm{Co}\left(\mathrm{NO}_{3}\right)_{2} \cdot 6 \mathrm{H}_{2} \mathrm{O}$ with $\mathrm{ZnO}$ also forming a hard cement during impregnation indicating the reactive synthsis over more conventional inert impregnation catalysts. This transition is completed at room temperature in a beaker, subsequent calcination of the material forms a green/black material for catalyst testing.

Table 1 Showing the catalyst metal loading and the surface area of the support materials used for the catalysts. The spinel crystallite size is taken from ex-situ XRD analysis

\begin{tabular}{llllll}
\hline $\begin{array}{l}\text { Support } \\
\text { material }\end{array}$ & Co wt\% & Mn wt\% & $\begin{array}{l}\text { Surface } \\
\text { area, } \\
\mathrm{m}^{2} / \mathrm{g}\end{array}$ & $\begin{array}{l}\text { XRF sul- } \\
\text { phur }\end{array}$ & $\begin{array}{l}\text { Cobalt } \\
\text { spinel size } \\
(\mathrm{\AA})\end{array}$ \\
\hline Titania & 10.8 & 1.22 & 54 & - & 58 \\
Ceria & 10.2 & 1.16 & 2.5 & - & 121 \\
Silica & 11.1 & 1.26 & 350 & 0.78 & 75 \\
$\gamma$-alumina & 10.8 & 1.22 & 222 & - & 51 \\
Zincite & 10.7 & 1.04 & 4.5 & - & 54 \\
Zirconia & 10.6 & 1.18 & 93 & - & 68
\end{tabular}




\subsection{In Situ XRD}

The in situ XRD allows for a temperature resolved study of the cobalt crystalline phases during the reduction of the oxide structures of cobalt to form metallic cobalt as shown in Eq. 7 [23]. Rietveld analysis of the individual diffraction patterns allows the quantification of the relative concentration of the individual phases (Fig. 9). Assessing the reduction in concentration of one phase, gives an indication of the reduction of this prior to the next phase being visible
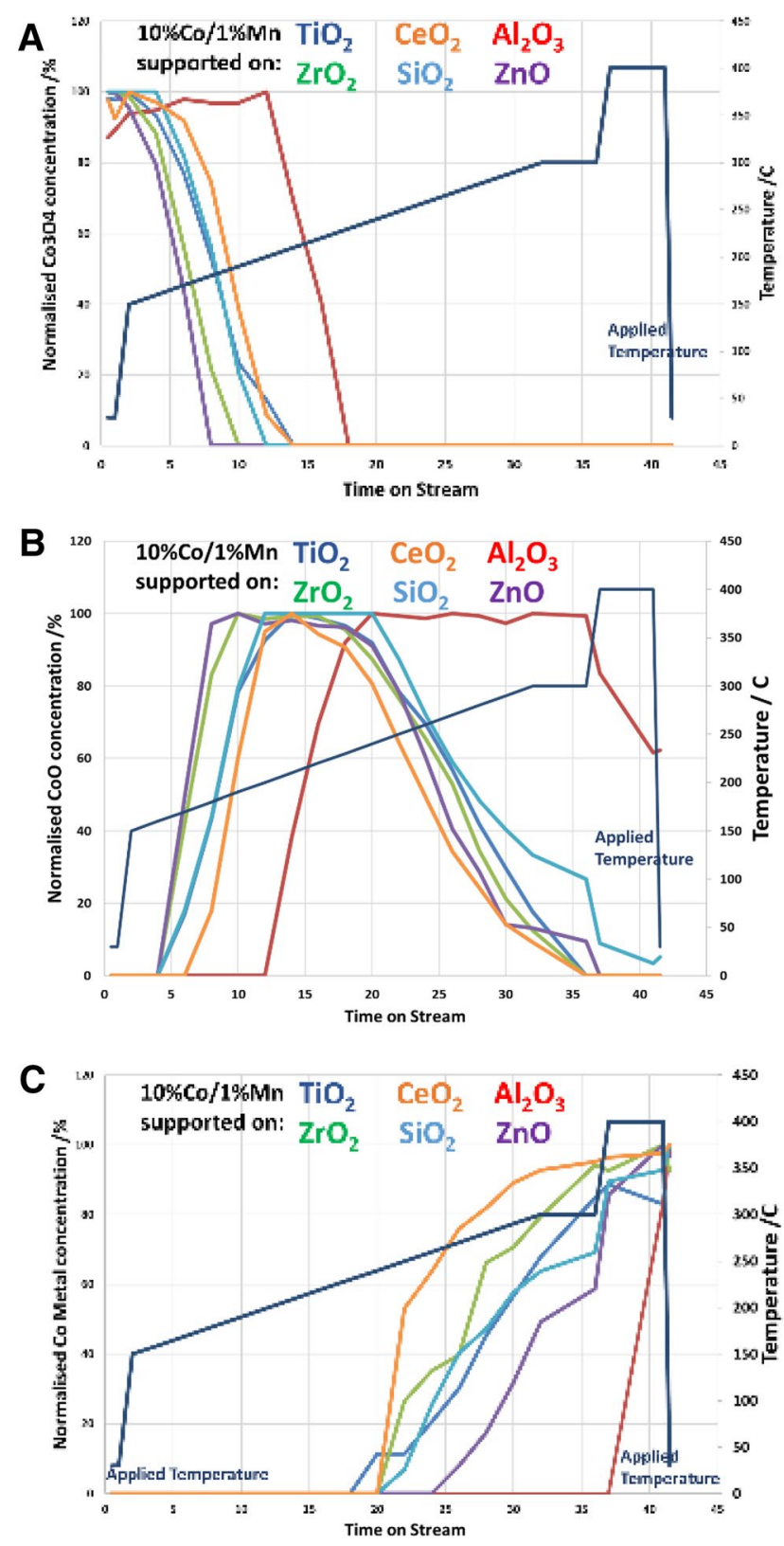

Fig. 9 The relative peak monoxide concentration of cobalt during the reduction by in situ XRD. Disappearance of $\mathrm{Co}_{3} \mathrm{O}_{4}(\mathbf{a})$, formation and loss of $\mathrm{CoO}(\mathbf{b})$ and growth of cobalt metal (c) during reduction in the diffraction pattern due to the initial low concentration or effectively very small crystallite sizes. Assessing the reduction in concentration of the monoxide peak proves to be more reliable for studying the formation of the metallic cobalt, rather than looking at the metallic cobalt peaks alone. This is due to the metallic cobalt forming with two different structures, with either a hexagonal close packed (HCP) structure or face centred cubic (FCC) structure. The cobalt structure formed is neither purely HCP or FCC, but an interlayered mixture of both due in part to the very similar energy of formation of the two phases [24, 25]. The stacking faults through the crystals result in comparatively broad or no diffraction peaks through those layers where stacking faults exist. This leaves a single peak to assess the metallic cobalt formation, so looking at the reduction of the monoxide structure provides more indicative peaks.

The reduction of the cobalt oxide species in the in situ XRD occurs at much lower temperatures than those observed in the TGA due to the much higher partial pressure
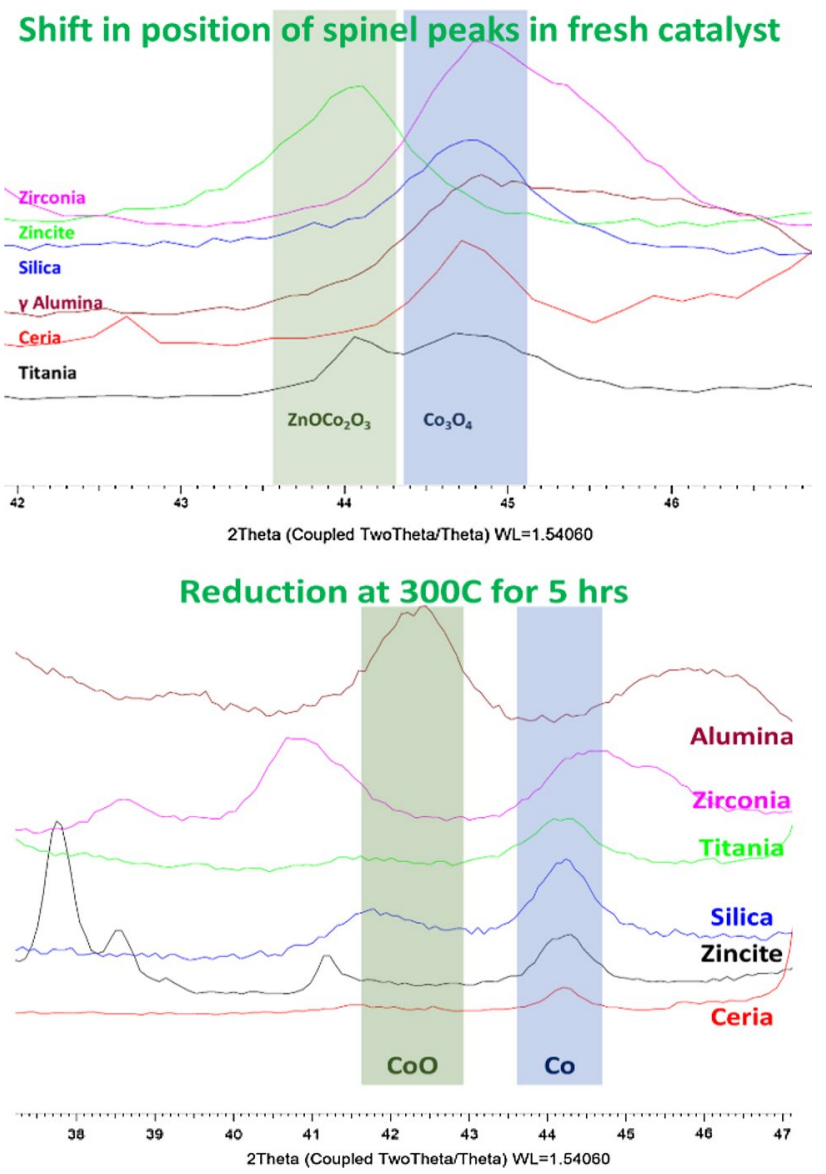

Fig. 10 Showing the difference between the spinel phases in a fresh catalyst where peaks in a zincite supported catalyst are shifted to significantly lower angle and the extent of reduction after $5 \mathrm{~h}$ at $300{ }^{\circ} \mathrm{C}$ under pure flowing hydrogen where alumina supported catalyst is still entirely $\mathrm{CoO}$ 
of hydrogen, where in the XRD this is 1 bar. However, the relationship between the steps of reduction and the relative order of reduction between both techniques is consistent. Generally, a higher temperature for the emergence of the monoxide structure in the diffraction patterns is mirrored with a higher temperature for the reduction of the concentration of the monoxide structure. Again, the exception to this is the ceria supported catalyst, where the emergence of the monoxide structure in the diffraction pattern is seen higher than the other catalysts except for the alumina supported catalyst. However, the reduction in the concentration of the monoxide occurs at the lowest temperature of this set of catalysts. Given the crystallite size of the cobalt spinel was $121 \AA$, compared with an average of around $60 \AA$ for the other catalysts, the larger crystallite size of the cobalt phases is easier to reduce and so the ceria supported catalysts are reducing at a lower temperature. The 1st stage of reduction is less susceptible but $\mathrm{CoO}$ to $\mathrm{Co}^{0}$ is more resistant to reduction for smaller cobalt particles [6].

The alumina sample is also significantly different from the other catalysts, as was the case in the TGA analysis. The cobalt monoxide in the alumina supported catalyst does not reduce in intensity at all at $300{ }^{\circ} \mathrm{C}$, whereas as shown in Fig. 10, all the catalysts exhibit metallic cobalt after $5 \mathrm{~h}$ at $300{ }^{\circ} \mathrm{C}$. The reduction of the cobalt monoxide phase is not seen until the sample was taken to $400{ }^{\circ} \mathrm{C}$ in the in situ $\mathrm{XRD}$, where the concentration of the monoxide was falling steadily.

The study of the reduction of the cobalt oxide phases on the silica supported catalyst, allows clearer visibility of the metallic cobalt structure which is not possible on catalysts on other supports where the peaks form the support overlap heavily with the cobalt phases. However, with amorphous silica as the support, all the cobalt phases may be observed clearly in the diffraction pattern. Here on the silica supported catalyst, following reduction of the cobalt oxide phases, the interlayered nature of the cobalt metal is observed. Cobalt may exist with either an HCP of an FCC structure, but typically not all the peaks expected from either structure can be seen in the diffraction pattern although those peaks are observed on the reduced silica supported catalyst, however not with the expected relative peak intensities (Fig. 11). Indeed, the highest intensity peak and also the sharpest is observed at $44.1^{\circ} 2 \theta$, which may be from the $\left[\begin{array}{lll}0 & 0 & 2\end{array}\right]$ peak in the HCP structure or the $\left[\begin{array}{lll}1 & 1 & 1\end{array}\right]$ peak in the FCC structure. Although when considering a hard sphere packing model, these would represent diffraction along the same layer. The diffraction peaks which would be generated though the layers when considering the hard sphere model, such as the $\left[\begin{array}{lll}1 & 0 & 1\end{array}\right]$ in the $\mathrm{HCP}$ at $47.2^{\circ} 2 \Theta$ and the $[200] \mathrm{FCC}$ peak at $51.4^{\circ} 2 \Theta$ are very low in intensity and much broader than the peak at $44.1^{\circ} 2 \Theta$. The measured relative peak intensity ratios with the background removed of the $44.1^{\circ}: 47.2^{\circ}: 51.4^{\circ}$ are approximately 10:3:2 where the expected ratio for [0 0 2]:[ $\left.\begin{array}{lll}1 & 0 & 1\end{array}\right]$ for HCP is approximately $1: 4$ and the expected ratio of [ $\left[\begin{array}{lll}1 & 1 & 1\end{array}\right]:\left[\begin{array}{lll}2 & 0 & 0\end{array}\right]$ for FCC is approximately 5:2. These expected ratios from the ICDD are significantly different to the observed relative intensity ratios of the measured peaks and cannot be fit by a physical mixture where both theoretical patterns are summed to give the observed pattern, suggesting a loss of intensity of diffraction peaks through the layers from the interlayering. Also, the peak broadening in these weaker intensity diffraction peaks supports the interlayering, as the peaks become broader from reduced long-range order.

The data in Table 2 highlights the shift in the unit cell size of the zincite supported catalyst and covers the changes in the unit cell size of the three different cobalt structure observed during the reduction. It is typically observed that the crystallite size falls slightly between the cobalt spinel phase and the cobalt monoxide phase. This is entirely expected as the removal of some of the oxygen from the crystals reduces the volume of the individual crystals too. For the subsequent reduction step, the crystallite size of the cobalt metal increases significantly. This would be what is expected should there be no sintering or rearrangement of the metallic cobalt. The results in Table 2 clearly show there is significant sintering of the cobalt during the final stage of reduction. Whilst there is water produced during the reduction of the oxide phases which is known to cause sintering during the reduction of the cobalt oxides, the measurement in the in situ XRD uses a very high GHSV and the diffraction measurement is only being carried out on the very top layer of the catalyst powder [6]. Because of this, the water concentration produced in the reduction will not be in high enough concentration to affect the sintering.

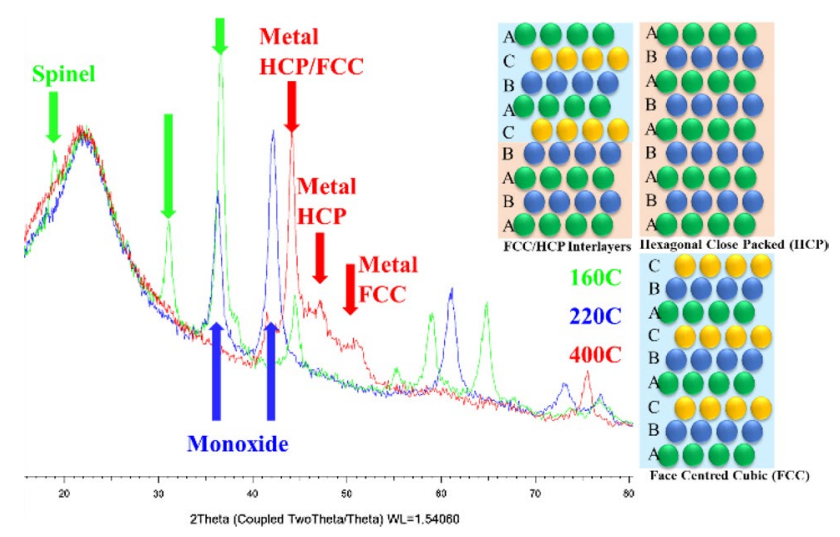

Fig. 11 Cobalt phases on $\mathrm{SiO}_{2}$ during reduction and the $\mathrm{HCP} \& \mathrm{FCC}$ peaks of fully reduced metal 
Table 2 Showing the unit cell dimensions of the cobalt spinel before the in situ XRD reduction and the crystallite sizes of the cobalt phases through the reduction. Spinel/CoO crystallite sizes were calculated by Rietveld refinement, $\mathrm{Co}^{0}$ metal used the Scherrer equation (using peak at $44^{\circ} 2 \Theta$ )

\begin{tabular}{lllll}
\hline $\begin{array}{l}\text { Catalyst }(10 \% \\
\text { Co, } 1 \% \mathrm{Mn})\end{array}$ & $\begin{array}{l}\mathrm{Co}_{3} \mathrm{O}_{4} \\
\text { Unit Cell, } \\
30 \mathrm{C}, \AA\end{array}$ & $\begin{array}{l}\mathrm{Co}_{3} \mathrm{O}_{4} \\
@ 150^{\circ} \mathrm{C}, \AA\end{array}$ & $\begin{array}{l}\mathrm{Co}(\mathrm{II}) \mathrm{O} \\
@ 210^{\circ} \mathrm{C}, \AA\end{array}$ & $\begin{array}{l}\mathrm{Co}^{(0)} \\
@ 300{ }^{\circ} \mathrm{C}, \AA\end{array}$ \\
\hline Titania & 8.108 & 59 & 49 & 108 \\
Ceria & 8.095 & 95 & 76 & 207 \\
Silica & 8.096 & 76 & 58 & 87 \\
$\gamma$-alumina & 8.081 & 60 & 34 & $\mathrm{~N} / \mathrm{A}$ \\
Zincite & 8.225 & 54 & 46 & 101 \\
Zirconia & 8.105 & 67 & 57 & 47 \\
\hline
\end{tabular}

\subsection{Catalysis Results}

Catalyst performance testing was carried out in a high throughput unit, with common gas feeds, temperatures and pressures (Fig. 12). Online GC analysis provided real time information on conversion and selectivity up to C8. The conditions for this were using a standard fixed bed reference rather than for CANS reactors. Given the catalysts are on different supports it can be difficult to optimise conditions for each. As such, they were all reduced at $300{ }^{\circ} \mathrm{C}$ in $100 \%$ hydrogen at high flow rates and were verified by XRD to achieve cobalt metal. Similarly, catalyst activity varied significantly across the catalysts and with reactor constraints (common flows/temperatures) different $\mathrm{CO}$ conversions were achieved. The silica supported catalyst was tested but due to the presence of sulphur (Table 1) it gave no meaningful FT activity but did provide a model system for characterization. Figure 13 shows conversion ranging from less than $10 \%$ to over $80 \%$, and the corresponding selectivity variations which are due in part to the conversion ranges. The catalyst supported on ceria showed low activity throughout, and this is in good agreement with the XRD data showing larger cobalt particles on the low surface area support. Zirconia and titania made considerably more active catalysts than alumina or zinc oxide.

By adjusting temperatures stepwise through the test, a comparison of performance at similar conversions could be achieved. Figure 13 shows the comparison of methane and $\mathrm{C}_{5+}$ selectivity at similar conversion.

Catalyst selectivity for olefins and alcohols (Fig. 14) has been reported previously as a new and exciting area of development for chemicals and base oils by FT [5, 26]. Ceria has often been demonstrated as a support for this due to its 'oxygen pool' from support reduction [27-29]. In this work ceria once again produced the highest level of oxygenates (predominantly alcohols and aldehydes), with $15 \%$ of the online product being functionalised in this way.

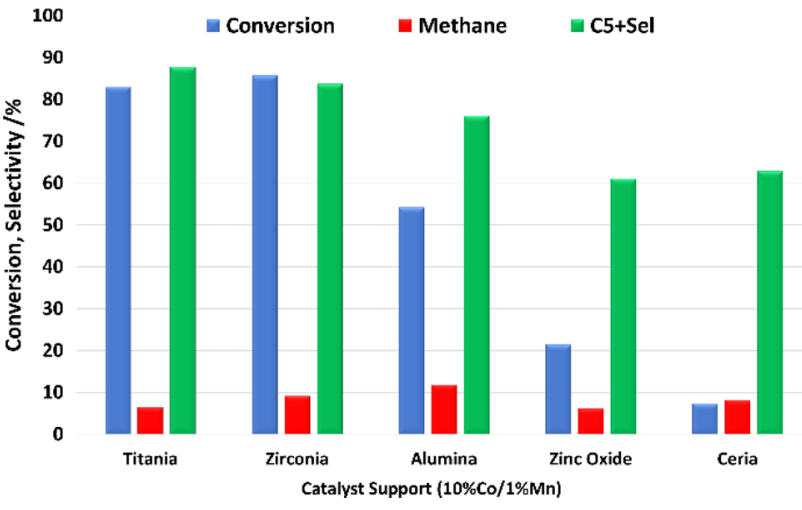

Fig. 12 Performance of a series of $10 \% \mathrm{Co} / 1 \% \mathrm{Mn}$ catalysts on different supports $\left(120-144 \mathrm{~h}, 245^{\circ} \mathrm{C}, 30 \mathrm{barg}, 1.8 \mathrm{H}_{2}: \mathrm{CO}, 3000 \mathrm{~h}^{-1}\right)$

Other partially reducible supports $\left(\mathrm{ZrO}_{2} / \mathrm{TiO}_{2}\right)$ showed little activity for oxygenate formation in these tests. Role of support in oxygenate formation is vital, and $\mathrm{ZnO} / \mathrm{CeO}_{2}$ are both noted for their defect sites and oxygen vacancies which are often used in catalysis and other applications such as semiconductors.

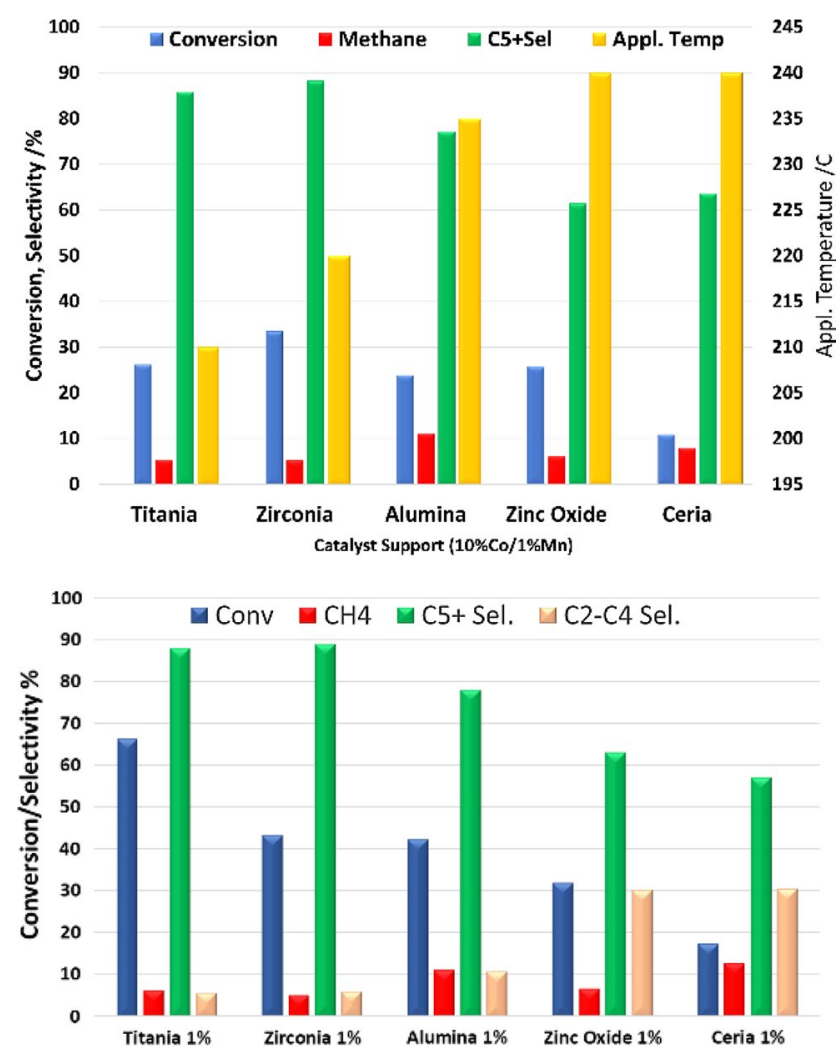

Fig. 13 Methane and $\mathrm{C}_{5+}$ Selectivity at similar conversions, by adjusting temperature (top) or adjusting catalyst mass (bottom) for $10 \% \mathrm{Co} 1 \% \mathrm{Mn}$ on different supports 


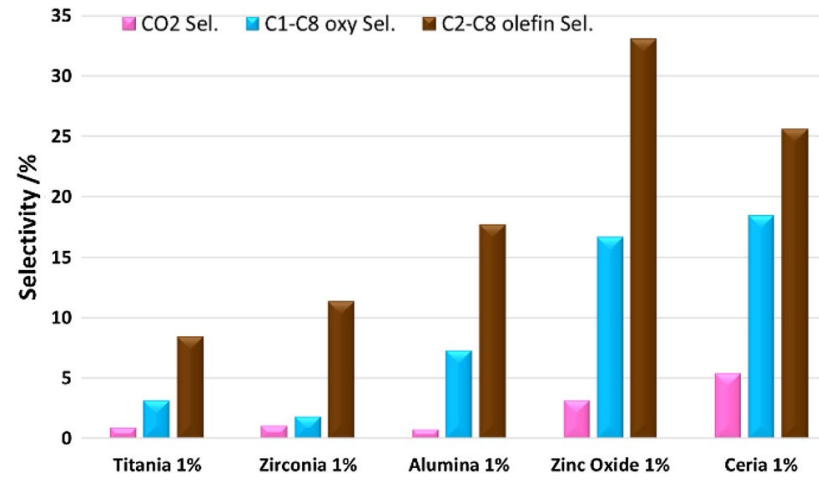

Fig. 14 - Selectivity for $\mathrm{CO}_{2}$, alcohols and olefins across different catalyst supports with $10 \% \mathrm{Co} 1 \% \mathrm{Mn}$ loading $\left(1.8 \mathrm{H}_{2}: \mathrm{CO} .30 \mathrm{barg}\right.$, $3000 \mathrm{~h}^{-1}, 20 \% \mathrm{~N}_{2}, 120-144 \mathrm{~h}$ )

\section{Conclusion}

A summary of the BP \& Johnson Matthey Fischer Tropsch technology is presented here, highlighting the benefits of the advanced CANS catalyst carrier reactor design and catalyst for improved performance. Superior heat transfer, reduced pressure drop and higher productivity lead to major economic savings. A fundamental understanding of the catalyst is also key to providing long term stable operation at scale. This includes focused evaluation of each stage of a catalyst life from synthesis, calcination, reduction, start-up, operation and deactivation. An example of this is shown by comparing catalysts on different supports using in situ X-ray diffraction during reduction.

The change in supports used between the different catalyst samples affects the temperatures of reduction of the cobalt oxide phases, although all catalysts other than the alumina-based sample will reach metallic cobalt at $300{ }^{\circ} \mathrm{C}$. However, the range of supports with the same cobalt loading do not produce a catalyst that looks or performs the same under catalytic testing. The very low surface area ceria supported catalyst produces much larger metal crystallite size. This reduces the metal surface area significantly and consequently contributes to the lower overall activity of this catalyst when compared to the well performing samples from a conversion perspective.

The largest differentiation in performance under standard fixed bed conditions comes from the support interaction rather than the physical properties of the cobalt. Whilst the large cobalt crystallites on the ceria supported catalyst results in low carbon monoxide conversion, the very small cobalt crystallites in the zirconia support catalyst increases the methane selectivity. This shows how the supports are influencing the overall performance of the catalysts. Both the titania and zirconia supported catalysts performed well, achieving high conversion with lower applied temperature. All the other catalysts require higher applied temperature to achieve good conversion. Furthermore, the changes to the catalyst support alter the distribution of the products, with the titania and zirconia supported catalysts producing predominantly paraffin products, whereas the change to ceria or zincite as the catalyst support produces significantly more olefins and alcohols showing the product distribution may be tailored depending on the support material.

Funding This study was funded by BP and Johnson Matthey through internal research and development programmes in Fischer-Tropsch.

\section{Compliance with Ethical Standards}

Conflict of interest The authors declare that they have no conflict of interest.

Open Access This article is licensed under a Creative Commons Attribution 4.0 International License, which permits use, sharing, adaptation, distribution and reproduction in any medium or format, as long as you give appropriate credit to the original author(s) and the source, provide a link to the Creative Commons licence, and indicate if changes were made. The images or other third party material in this article are included in the article's Creative Commons licence, unless indicated otherwise in a credit line to the material. If material is not included in the article's Creative Commons licence and your intended use is not permitted by statutory regulation or exceeds the permitted use, you will need to obtain permission directly from the copyright holder. To view a copy of this licence, visit http://creativecommons.org/licenses/by/4.0/.

\section{References}

1. European Commission: Renewable Energy 2018 https://ec.europ a.eu/energy/en/topics/renewable-energy

2. Senecal P, Jacques SDM, Di Michiel M, Kimber SAJ, Vamvakeros A, Odarchenko Y, Lezcano-Gonzalez I, Paterson J, Ferguson E, Beale AM (2017) ACS Catal 7:2284-2293

3. Beale AM, Jacques SDM, Di Michiel M, Mosselmans JFW, Price SWT, Senecal P, Vamvakeros A, Paterson J (2018) Philos Trans Soc A 376:20170057

4. Puga AV (2018) Catal Sci Technol 8:5681

5. Paterson J, Peacock M, Purves R, Partington R, Sullivan K, Sunley G, Wilson J (2018) ChemCatChem 10:5154-5163

6. Paterson J, Peacock M, Ferguson E, Ojeda M, Clarkson J (2017) Appl Catal A 546:103-110

7. Wolf M, Fischer N, Claeys M (2016) Catal Today 275:135-140

8. Fratalocchi L, Groppi G, Visconti CG, Lietti L, Tronconi E (2019) Catal Today 342:79-87

9. Paterson J, Peacock M, Ferguson E, Purves R, Ojeda M (2017) ChemCatChem 9:3463-3469

10. Bartholomew CH (2001) Appl Catal A 212:17-60

11. Claeys M, Dry ME, van Steen E, du Plessis E, van Berge PJ, Saib AM, Moodley DJ (2014) J Catal 318:193-202

12. Wolf M, Gibson EK, Olivier EJ, Neethling JH, Catlow CRA, Fischer N, Claeys M (2019) ACS Catal 9:4902

13. Okoye-Chine CG, Moyo M, Liu X, Hildebrandt D (2019) Fuel Process Technol 192:105-129

14. Rytter E, Holmen A (2015) Catalysts 5:478 
15. Font Freide JJHM, Gamlin TD, Graham C, Hensman JR, Nay B, Sharp C (2003) Top Catal 26:3-12

16. Font Freide, J. J. H. M., Collins, J. P., Nay, B., and Sharp, C. (2007), In: Davis BH, Occelli ML (Eds) Studies in surface science and catalysis, Elsevier, Amsterdam, pp 37-44

17. Coe A, Paterson J (2019) Chem Eng 937:30-34

18. BP Press Office (2018) BP and Johnson Matthey license innovative waste-to-fuels technology to biofuels producer Fulcrum BioEnergy, BP Press Office. https://www.bp.com/en/global/corporate/ media/press-releases/bp-and-johnson-matthey-license-innovative -waste-to-fuels-technology-to-biofuels-producer-fulcrum-bioen ergy.html

19. Bezemer GL, Bitter JH, Kuipers HPCE, Oosterbeek H, Holewijn JE, Xu X, Kapteijn F, van Dillen AJ, de Jong KP (2006) JACS 128:3956-3964

20. Liu J-X, Wang P, Xu W, Hensen EJM (2017) Engineering 3:467-476

21. Gamlin TD (2014) US8906970 (B2), Davy Process Tech LTD

22. Perry NH, Mason TO, Ma C, Navrotsky A, Shi Y, Bettinger JS, Toney MF, Paudel TR, Lany S, Zunger A (2012) J Solid State Chem 190:143-149

23. Garces LJ, Hincapie B, Zerger R, Suib SL (2015) J Phys Chem C 119:5484-5490
24. Velterop L, Delhez R, de Keijser TH, Mittemeijer EJ, Reefman D (2000) J Appl Cryst 33:296-306

25. Price SWT, Martin DJ, Parsons AD, Sławiński WA, Vamvakeros A, Keylock SJ, Beale AM, Mosselmans JFW (2017) Sci Adv 3:e1602838

26. Xie J, Paalanen PP, van Deelen TW, Weckhuysen BM, Louwerse MJ, de Jong KP (2019) Nat Commun 10:167

27. Riberio MC, Gnanamani MK, Azevedo IR, Rabelo-Neto RC, Pendyala VRR, Jacobs G, Davis BH, Noronha FB (2014) Am Chem Soc Div Energy Fuels 59:797-799

28. Gnanamani MK, Jacobs G, Shafer WD, Martinelli M, Cronauer DC, Kropf AJ, Marshall CL, Davis BH (2017) Appl Catal A 547:115-123

29. Gnanamani MK, Jacobs G, Graham UM, Ribeiro MC, Noronha FB, Shafer WD, Davis BH (2016) Catal Today 261:40-47

Publisher's Note Springer Nature remains neutral with regard to jurisdictional claims in published maps and institutional affiliations. 